\title{
Microscale Automated Alignment and Spatial Tracking Through Structured Illumination
}

\author{
Mark Stonehouse, Alex Blanchard, Benoit Guilhabert, Ian Watson, Erdan Gu, \\ Johannes Herrnsdorf, and Martin Dawson \\ Institute of Photonics, Department of Physics, University of Strathclyde, Glasgow G1 1RD (UK), \\ Email: mark.stonehouse@ strath.ac.uk
}

\begin{abstract}
We present a tracking and alignment capability for use in mask-less lithography systems. Through the use of micropixellated light emitting diode (LED) arrays, fluorescent clusters can be identified and used as alignment markers at micrometer scale.
\end{abstract}

\section{INTRODUCTION}

Automation and control is the driving force behind a modern day industrial revolution [1]. Through the use of sensors and feedback mechanisms, new automated manufacturing processes are able to adapt to situations that cannot practically be dealt with using current technology. It has been shown previously that micro-scale LEDs ( $\mu$ LEDs) can be used in maskless lithography to define photoresist features with submicron precision [2], an approach that will benefit strongly from adaptive automation.

We describe here the incorporation of an intelligent alignment function into such a direct-writing optical lithography setup. This is achieved through structured illumination wherein we are able to spatially locate and track designated features [3]. Spatial tracking and photocuring is done by using two separate $\mu$ LED arrays at different wavelengths, with $405 \mathrm{~nm}$ used for photocuring and $510 \mathrm{~nm}$ for structured illumination. The positioning capability is demonstrated on the example of red quantum dot (QD) clusters which fluoresce under excitation by the green/blue light emitted by the LED arrays. These clusters are distributed randomly, thus highlighting the capability of the system to adapt to elements that do not have accurate position control in earlier manufacturing stages.

In this report we discuss the implementation of structured illumination functionality within the system and the control mechanisms behind it.

\section{Structured Light PRINCIPLE}

In so-called 'structured illumination', the LED array is projected onto the sample to create a chess board style grid, where each grid site is illuminated by one LED element [3]. The array then sends a series of purpose-designed illumination patterns that are chosen such that each LED emits a unique modulation 'fingerprint'. In earlier work, this fingerprint was sensed by active receivers in the area [3]. Instead, we exploit here the fact that a reflective or fluorescent marker located at one grid site will relay the corresponding fingerprint signal to a remote optical detector, which could be a camera, spectrometer or a single-photon sensitive detector. This way, the position of a marker within the FOV can be tracked using the pattern projection by the LED array and remote detection. The insets of Figs. 1 and 3 show representative patterns of the illumination pattern sequence used here, including the red fluorescence signal that relays the local optical signal. Fig. 1 shows a block diagram of the process, indicating pattern projection onto the sample, remote detection, spectral filtering to isolate the relayed local signal, and feedback of the retrieved position to the motorized sample stage.

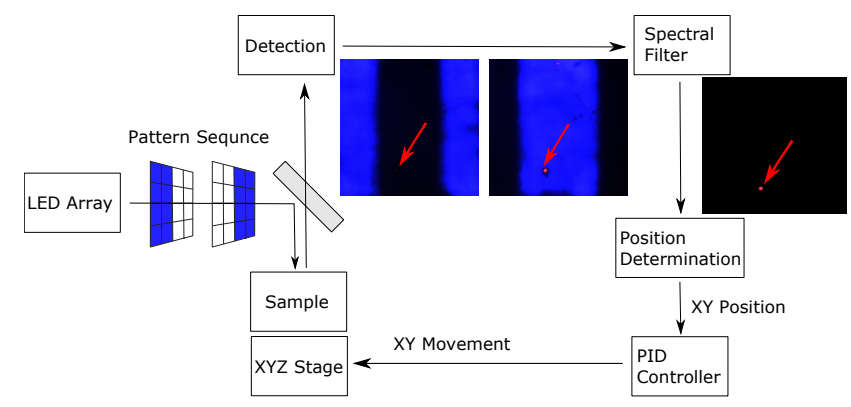

Figure 1. A schematic diagram showing how the pattern sequence is analyzed

\section{OpticAl SETUP}

A schematic diagram of the optical system is shown in Fig. 2. An earlier version of this has been previously reported [4], though there have subsquently been substantial additions in both the mechanical parts and the driving software. Here the light from each array is collimated with an aspheric lens (f3,4) before being added to the main optical path with dichroic mirrors (D1,2). The light then passes through two spherical lenses $(\mathrm{f} 1,2)$ to adjust the spot size before being reflected off a beam splitter (BS) into a microscope objective (10X) to be focused onto our sample. The sample is observed with a CCD camera using a tube lens (TL) and mirror (M) to focus the light. The camera operates at triple the frame rate of the LED arrays (12 fps) in order to remove the need for synchronization. Control of both the $\mu \mathrm{LED}$ array, as well as the motorized $\mathrm{XYZ}$ stage is achieved through a MATLAB ${ }^{\circledR}$ interface which allows for each device to be modulated independently. Whilst in operation, the structured light elements continuously cycle through a sequence as discussed which allows for the position of a reflective or fluorescent marker to be tracked live at a rate currently limited by the computer's processing speed 
and the overhead due to asynchronous acquisition. An RGB camera was used here to enable independent monitoring of the alignment process as well as coarse spectral filtering. This could be replaced by a single spectrometer, which would retain the alignment capability and offer highly versatile spectral filtering.

The sample is moved by a motorized XYZ stage which is controlled through a feedback loop based on the positioning code. The stage itself uses pico-motors with a step size of 0.5 $\mu \mathrm{m}$ which require external control for precise re-positioning. To counter this, and improve accuracy even if the motors are improved, a PID controller was implemented which takes the samples current position into consideration when moving the sample. The overall accuracy of locating the marker is currently limited by the spot size projected by the LED array.

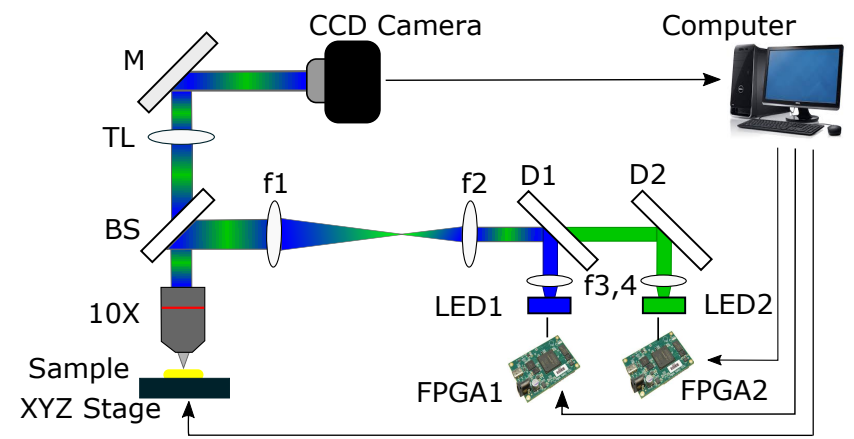

Figure 2. A schematic of the optical setup

\section{RESUlts}

For this demonstration, a $20 \mu \mathrm{m}$ diameter cluster of redemitting QDs is used as the alignment target.

Typically the stage needs only two cycles of XY movement to be at its final location though this is dependent on how far the stage has to travel and the inaccuracies in that specific movement. From one corner to another the most observed is three cycles. A representative trajectory of a QD cluster being aligned to a target position is shown in Fig. 3. In this test we aligned the marker to coordinates $(13,11)$ and set a target of $(15,15)$, where each grid coordinate is $\approx 20 \mu \mathrm{m}$ apart. The final position was successfully within the correct pixel and took two stage movements to finalize its position. This shows that any light which does not interfere with either the photoresist or overlap with the QD cluster emission can be used. It also shows that even though the LED array has only $41 \%$ fill factor this does not compromise the alignment function.

\section{CONCLUSiON}

We have implemented the ability to autonomously locate, track and align fluorescent QD clusters with micro-meter precision within an LED-based mask-less photo-lithography setup. This was achieved with both blue and green light though is not limited to this. We have proven that by using structured illumination we can add layers of additional functionality onto existing technology allowing for the potential improvement of existing manufacturing processes.
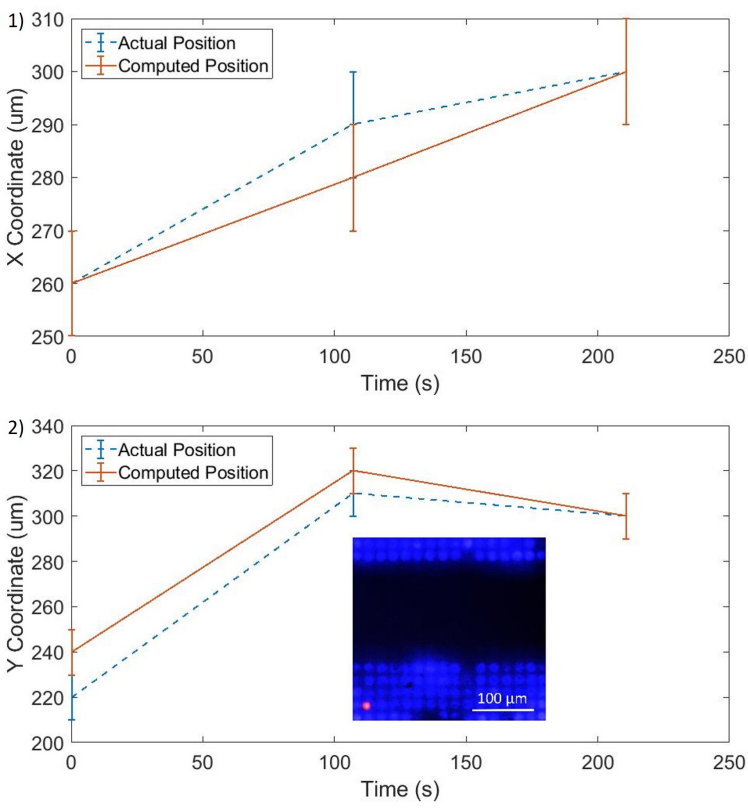

Figure 3. Trajectory of a QD cluster during one illustrative alignment process Shown are the actual position of the cluster as monitored by the camera and the position computed by the alignment algorithm. Error bars indicate the alignment grid resolution. (1) shows the $\mathrm{x}$-coordinate, and (2) the y-coordinate as a function of time. Inset: representative camera frame.

\section{ACKNOWLEDGMENT}

Supporting data are funded through EPSRC under grant no. EP/P02744X/2 and EP/S001751/1. Additional thanks to Pedro Alves and Nicolas Laurand for supplying the QD clusters. Data is available at: https://doi.org/10.15129/7cf60f24-06784818-a017-719bb75f363c

\section{REFERENCES}

[1] Heiner Lasi, Peter Fettke, Hans-Georg Kemper, et al. "Industry 4.0". In: Business \& information systems engineering 6.4 (2014), pp. 239-242.

[2] Benoit Guilhabert, David Massoubre, Elliot Richardson, et al. "Sub-micron lithography using InGaN micro-LEDs: mask-free fabrication of LED arrays". In: IEEE Photonics Technology Letters 24.24 (2012), pp. 2221-2224.

[3] Johannes Herrnsdorf, Martin D Dawson, and Michael J Strain. "Positioning and Data Broadcasting Using Illumination Pattern Sequences Displayed by LED Arrays". In: IEEE Transactions on Communications 66.11 (2018), pp. 5582-5592.

[4] Mark Stonehouse, Yanchao Zhang, Benoit Guilhabert, et al. "Digital illumination in microscale direct-writing photolithography: challenges and trade-off'. In: IEEE British and Irish Conference on Optics and Photonics. 2018. 